Relativistic electron microbursts due to nonlinear pitch angle scattering by EMIC triggered emissions

$\operatorname{AUTHOR}(S):$

Omura, Yoshiharu; Zhao, Qinghua

CITATION:

Omura, Yoshiharu ...[et al]. Relativistic electron microbursts due to nonlinear pitch angle scattering by EMIC triggered emissions. Journal of Geophysical Research: Space Physics 2013, 118(8): 5008-5020

ISSUE DATE:

2013-08

URL:

http://hdl.handle.net/2433/192997

RIGHT:

(C)2013. American Geophysical Union. 


\title{
Relativistic electron microbursts due to nonlinear pitch angle scattering by EMIC triggered emissions
}

\author{
Yoshiharu Omura ${ }^{1}$ and Qinghua Zhao ${ }^{1}$ \\ Received 17 May 2013; revised 5 July 2013; accepted 28 July 2013; published 13 August 2013.
}

[1] We show that the anomalous cyclotron resonance between relativistic electrons and electromagnetic ion cyclotron (EMIC) triggered emissions takes place very effectively near the magnetic equator because of the variation of the ambient magnetic field. Efficient precipitations are caused by nonlinear trapping of relativistic electrons by

electromagnetic wave potentials formed by EMIC triggered emissions. We derive the necessary conditions of the wave amplitude, kinetic energies, and pitch angles that must be satisfied for the nonlinear wave trapping. We have conducted test particle simulations with a large number of relativistic electrons trapped by a parabolic magnetic field near the magnetic equator. In the presence of coherent EMIC-triggered emissions with increasing frequencies, a substantial amount of relativistic electrons is trapped by the wave, and the relativistic electrons at high pitch angles are guided to lower pitch angles within a short time scale much less than a second, resulting in rapid precipitation of relativistic electrons or relativistic electron microbursts.

Citation: Omura, Y., and Q. Zhao (2013), Relativistic electron microbursts due to nonlinear pitch angle scattering by EMIC triggered emissions, J. Geophys. Res. Space Physics, 118, 5008-5020, doi:10.1002/jgra.50477.

\section{Introduction}

[2] Coherent emissions of electromagnetic ion cyclotron (EMIC) waves have been found in observations by the Cluster spacecraft in the magnetosphere [Pickett et al., 2010; Grison et al., 2013]. Most of the emissions show dynamic spectra with rising-tone frequencies triggered by constant frequency EMIC waves, and they are called EMIC triggered emissions. Since the characteristics of the emissions are very similar to those of whistler-mode chorus emissions [e.g., Tsurutani and Smith, 1974; Anderson and Kurth, 1989; Lauben et al., 1998, 2002; Santolik et al., 2003; Kasahara et al., 2009], a nonlinear theory, which is essentially the same as the nonlinear wave growth theory for whistler-mode chorus emissions [Omura et al., 2008, 2009], has been developed based on formation of electromagnetic proton holes in the velocity phase space [Omura et al., 2010]. The theory has been tested with the observations and simulations [Shoji and Omura, 2011, 2012; Shoji et al., 2011], finding good agreements in the nonlinear growth rates and the amplitude thresholds for the wave growth. These EMIC triggered emissions consisting of a series of rising tones are excited near the magnetic equator by energetic protons from several $\mathrm{keV}$ to a few hundred $\mathrm{keV}$ injected into the inner magnetosphere.

\footnotetext{
${ }^{1}$ Research Institute for Sustainable Humanosphere, Kyoto University, Kyoto, Japan.

Corresponding author: Y. Omura, Research Institute for Sustainable Humanosphere, Kyoto University, Uji, Kyoto, 611-0011, Japan. (omura@rish.kyoto-u.ac.jp)

(C)2013. American Geophysical Union. All Rights Reserved. 2169-9380/13/10.1002/jgra.50477
}

[3] In the generation process of EMIC triggered emissions, a substantial amount of the energetic protons are scattered into the loss cone [Shoji and Omura, 2011]. It has also been recognized well that EMIC waves can interact with relativistic electrons through the anomalous cyclotron resonance, and quantitative evaluation of the relativistic electron precipitation (REP) has been made based on the quasilinear diffusion model [e.g., Summers et al., 2007a, 2007b; Jordanova et al., 2008]. Ground and satellite observations show that EMIC waves cause precipitation of ions with energies of tens of $\mathrm{keV}$ and precipitation of relativistic electrons into an isolated proton aurora at the same time [Miyoshi et al., 2008; Spasojevic et al., 2011]. The Finnish pulsation magnetometer chain and riometer chain also confirmed the link between the EMIC waves and intense REP [Rodger et al., 2008]. Large losses of relativistic electrons due to EMIC waves are also observed at the recovery phases of geomagnetic storms [Sandanger et al., 2009]. A statistical study on EMIC-driven REP has been made by Carson et al. [2013], and they found that majority of proton precipitation associated REP occurred outside the plasmasphere.

[4] A theoretical and numerical analysis of nonlinear interaction between an EMIC wave and relativistic electrons was performed by Albert and Bortnik [2009] and Liu et al. [2012]. The study has been extended for a coherent EMIC wave with a variable frequency for application to EMIC triggered emissions by Omura and Zhao [2012] (hereinafter OZ12). In OZ12, the nonlinear trapping of resonant electrons results in very efficient pitch angle scattering due to combination of the rising frequency and the inhomogeneous magnetic field near the magnetic equator. The untrapped resonant electrons are scattered to higher pitch angles, while the trapped resonant electrons are transported to much lower 
pitch angles. Some of the electrons are scattered into the loss cone within a short time scale much less than a second. The rapid precipitations could be related to observations of relativistic electron microbursts at low altitudes [Lorentzen et al., 2001; Johnston and Anderson, 2010].

[5] We study the nonlinear resonant interaction to evaluate the efficiency of precipitation into the loss cone by the EMIC triggered emissions. The anomalous cyclotron resonance condition for different energies of electrons with different wave frequencies, which was not studied sufficiently in OZ12, is further analyzed in section 2 . We make use of the second-order resonance condition derived by OZ12 to find the amplitude threshold for the nonlinear trapping of electrons. In section 3, we examine the resonance conditions by test particle simulations assuming a simplified model of a wave packet used in OZ12. Implementing the frequency variation of an EMIC triggered emission based on the EMIC chorus equations [Omura et al., 2010], we perform test particle simulations with a large number of particles to find out rates of REP in section 4 . We present the summary and discussion in section 5 .

\section{Nonlinear Resonant Trapping of Relativistic Electrons by EMIC Triggered Emissions}

[6] An L-mode EMIC wave with a frequency $\omega$ and a wave number $k$ can interact with a relativistic electron satisfying the anomalous cyclotron resonance condition

$$
\omega-k v_{\|}=-\frac{\Omega_{e}}{\gamma},
$$

where $\gamma$ is the Lorentz factor of the electron. The electron cyclotron frequency is given by

$$
\Omega_{e}=\frac{e B_{0}}{m_{e}},
$$

where $e(>0), m_{e}$, and $B_{0}$ are the electron charge, rest mass, and the magnitude of the magnetic field, respectively. Based on the resonance condition, we define the resonance velocity as

$$
V_{R}=\frac{1}{k}\left(\omega+\frac{\Omega_{e}}{\gamma}\right)
$$

The frequency of the L-mode EMIC wave is below the proton cyclotron frequency $\Omega_{H}$. Since $\omega<\Omega_{H}<<\Omega_{e}$, we can approximate $V_{R}$ as

$$
V_{R}=\frac{\Omega_{e}}{\gamma k} .
$$

The linear dispersion relation of the EMIC wave [Omura et al., 2010] yields

$$
k=\frac{\sqrt{\omega \Pi_{c}}}{c},
$$

where $c$ is the speed of light, and $\Pi_{c}$ is given by (OZ12)

$$
\Pi_{c}=\omega \sum_{s} \frac{\omega_{p s}^{2}}{\Omega_{s}\left(\Omega_{s}-\omega\right)} .
$$

The variables $\Omega_{s}$ and $\omega_{p s}$ are the cyclotron frequency and the plasma frequency of ion species "s", respectively. In the present study, we assume presence of proton $\mathrm{H}^{+}$, helium $\mathrm{He}^{+}$, and oxygen $\mathrm{O}^{+}$ions. We assume the variation of

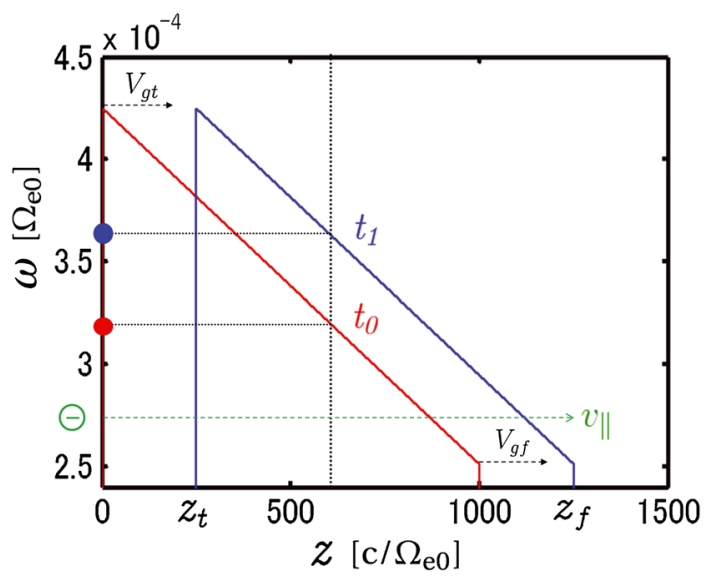

Figure 1. Schematic illustration of frequency variation of the EMIC wave packet at the initial time $t_{0}$ and a later time $t_{1}\left(>t_{0}\right)$ in red and blue, respectively. A resonant electron passes through the wave packet with $v_{\|}$much greater than the group velocities of the wave front $\left(V_{g f}\right)$ and tail $\left(V_{g t}\right)$. The position $z=0$ refers to the magnetic equator. (After OZ12).

the magnetic field near the equator is approximated by a parabolic function expressed by

$$
B_{0}(z)=B_{\mathrm{EQ}}\left(1+a z^{2}\right) \text {, }
$$

where $B_{\mathrm{EQ}}$ is the magnetic field at the equator, and $a=$ $4.5 /\left(L R_{E}\right)^{2}$. In the present study, we assume $a=3 \times 10^{-7}$ $\Omega_{e 0}^{2} / c^{2}$, which is the typical value at $L=4.27$. We normalize a time $t$ and a distance $z$ by $\Omega_{e 0}^{-1}$ and $c / \Omega_{e 0}$, respectively, where $\Omega_{e 0}$ is the electron cyclotron frequency at the magnetic equator. We also assume that the densities of ions vary proportionally with the intensity of the ambient magnetic field, i.e., $\omega_{p s}^{2} \propto B_{0}(z)$.

[7] The wave frequency varies as a function of a time $t$ and a position $z$. When a resonant electron interacts with a wave packet of an EMIC rising-tone emission, it goes through the wave packet with a parallel velocity $v_{\|}$much faster than the group velocity $\left(v_{\|} \gg V_{g}\right)$ of the packet. As schematically illustrated in Figure 1, the wave frequency $\omega$ seen by an electron moving away from the equator decreases, while the cyclotron frequency $\Omega_{s}$ increases as the magnitude of the magnetic field increases. Because of these variations, the wave number $k$ and the resonance velocity $V_{R}$ take wide ranges of values.

[8] As demonstrated by OZ12, the kinetic energy of a resonant electron hardly changes through interaction with EMIC waves (see OZ12, Figure 8). For simplicity, we assume the magnitude of the electron velocity is constant as $v_{0}$, which is expressed in terms of the Lorentz factor $\gamma$ as

$$
v_{0}=c \sqrt{1-\frac{1}{\gamma^{2}}} .
$$

Using the pitch angle $\alpha$ of the velocity, we can express the parallel velocity of the electron as

$$
v_{\|}=\frac{c}{\gamma} \sqrt{\gamma^{2}-1} \cos \alpha
$$




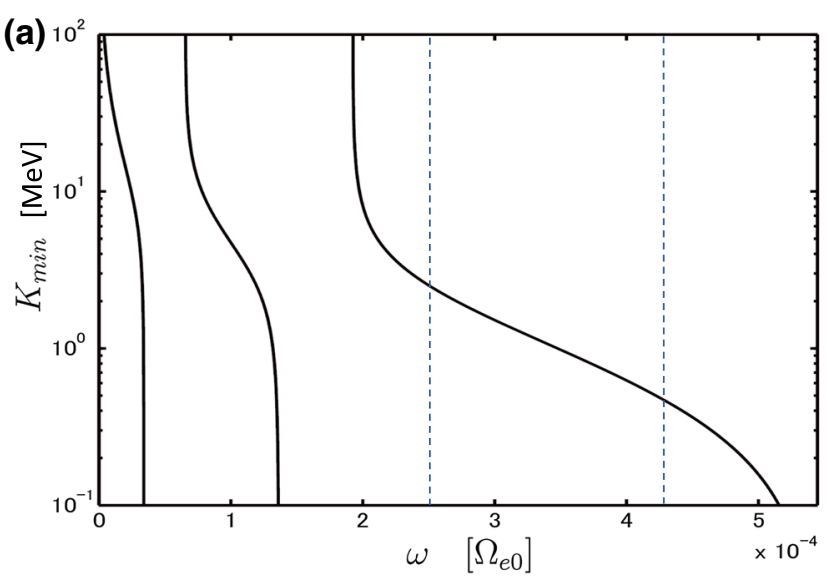

(b)

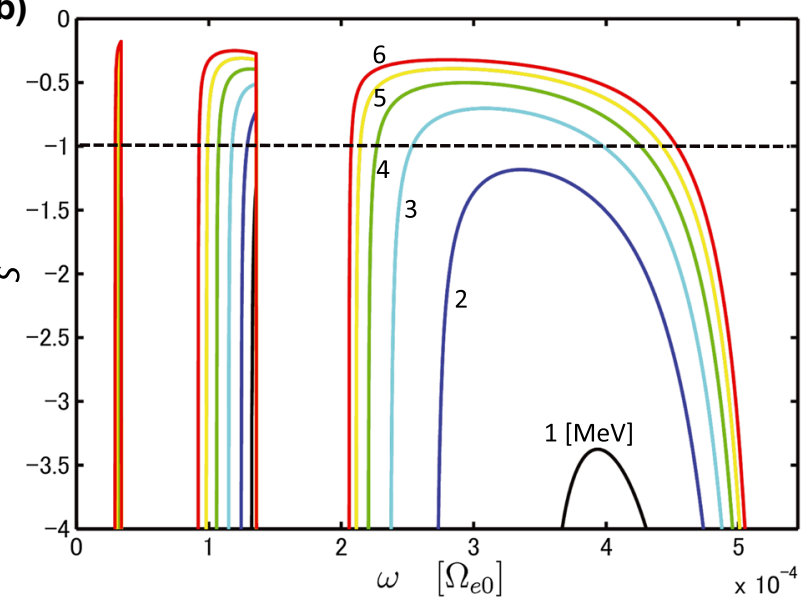

Figure 2. (a) Minimum resonance energy $K_{\min }$ as a function of the wave frequency $\omega$ at the magnetic equator $(z=0)$. The right edge of the $\omega$ axis is $\Omega_{H}(3.7 \mathrm{~Hz})$. Since we have oxygen and helium, ions in addition to protons, there exist three L-mode EMIC wave bands, i.e., oxygen, helium, and proton bands. The lower and upper limit of the wave frequency of the EMIC triggered emission is indicated by blue dashed lines. (b) Inhomogeneity factors $S$ at the equator for electrons of $1 \mathrm{MeV}$ (black), $2 \mathrm{MeV}$ (blue), $3 \mathrm{MeV}$ (cyan), $4 \mathrm{MeV}$ (green), $5 \mathrm{MeV}$ (yellow), and $6 \mathrm{MeV}$ (red).
Assuming the cyclotron resonance condition $v_{\|}=V_{R}$, we obtain from (4), (5), and (9)

$$
\cos \alpha=\frac{\Omega_{e}}{\sqrt{\omega \Pi_{c}\left(\gamma^{2}-1\right)}} .
$$

This equation is equivalent to the cyclotron resonance condition (1). Since electrons are scattered to low pitch angles close to $0^{\circ}$ through the resonance, $\cos \alpha$ approaches to unity. Substituting (10) into $\cos \alpha<1$, we have

$$
\gamma>\sqrt{1+\frac{\Omega_{e}^{2}}{\omega \Pi_{c}}} .
$$

Noting that $\gamma$ gives the kinetic energy $K=m_{e} c^{2}(\gamma-1)$, we can obtain an expression for the minimum resonance energy $K_{\min }$ as

$$
K_{\min }=m_{e} c^{2}\left(\sqrt{1+\frac{\Omega_{e}^{2}}{\omega \Pi_{c}}}-1\right)
$$

which is plotted in Figure 2a for the physical parameters listed in Table 1. We note that equations (42)-(46) of OZ12 concerning the derivation of the minimum kinetic energy are not exact because of the inappropriate assumption of an independent $v_{\perp}$, which is actually determined from the resonance condition $v_{\|}=V_{R}$. From (4), (5), and (8), we can obtain

$$
v_{\perp}=\frac{c}{\gamma} \sqrt{\gamma^{2}-1-\frac{\Omega_{e}^{2}}{\omega \Pi_{c}}},
$$

where $\gamma$ should satisfy (11). We make use of (13) in deriving conditions of nonlinear trapping of resonant electrons, and the evaluation of the inhomogeneity factor $S$ is given in Figure $2 b$ for comparison with Figure $2 a$. The frequency ranges of $S>-1$ correspond to the ranges of nonlinear trapping, which is described later.

[9] Assuming the parabolic variation of the ambient magnetic field, we plot pitch angles $\alpha$ given by (10) for five equi-spaced frequencies from $1.7 \mathrm{~Hz}\left(\omega=2.51 \times 10^{-4} \Omega_{e 0}\right.$, $\left.0.46 \Omega_{H 0}\right)$, where $\Omega_{H 0}$ is the proton cyclotron frequency at the equator, to $2.8 \mathrm{~Hz}\left(\omega=4.25 \times 10^{-4} \Omega_{e 0}, 0.76 \Omega_{H 0}\right)$ as functions of $z$ in blue, cyan, green yellow, and red, respectively in each panel of Figure 3. These curves represent the cyclotron resonance condition for different frequencies.

Table 1. Input Parameters

\begin{tabular}{lccc}
\hline Parameter & & Normalized Value & Real Value \\
\hline time step & $\Delta t$ & $0.2 / \Omega_{e 0}$ & $4.7 \times 10^{-6} \mathrm{~s}$ \\
grid spacing & $\Delta z$ & $1.0 \mathrm{c} / \Omega_{e 0}$ & $7.0 \mathrm{~km}$ \\
electron cyclotron frequency at equator & $f_{c e}$ & $6.8 \mathrm{kHz}$ \\
proton cyclotron frequency at equator & $f_{c H}$ & $3.7 \mathrm{~Hz}$ \\
electron plasma frequency at equator & $f_{p e}$ & $18 f_{c e}$ & $120 \mathrm{kHz}$ \\
electron density at equator & $n_{e}$ & & $178 / \mathrm{cc}$ \\
proton density at equator & $n_{H}$ & $0.81 n_{e}$ & $144 / \mathrm{cc}$ \\
helium density at equator & $n_{H e}$ & $0.095 n_{e}$ & $17 / \mathrm{cc}$ \\
oxygen density at equator & $n_{O}$ & $0.095 n_{e}$ & $17 / \mathrm{cc}$ \\
electron plasma frequency at equator & $\omega_{p e}$ & $18 \Omega_{e 0}$ & $7.5 \times 10^{5} \mathrm{rad} / \mathrm{s}$ \\
coefficient of parabolic magnetic field & $a$ & $3.0 \times 10^{-7} \Omega_{e 0}^{2} / c^{2}$ & $1.7-2.8 \mathrm{~Hz}$ \\
wave frequency & $f$ & $0.46-0.76 f_{c H}$ & $2.2 \mathrm{nT}$ \\
wave amplitude & $B_{w}$ & $0.009 B_{\mathrm{EQ}}$ & $800 \mathrm{~km} / \mathrm{s}$ \\
perpendicular velocity of energetic protons & $V_{\perp 0}$ & $0.0027 c$ & \\
\hline
\end{tabular}


OMURA AND ZHAO: RELATIVISTIC MICROBURSTS BY EMIC WAVES

(a) $1.1 \mathrm{MeV}$

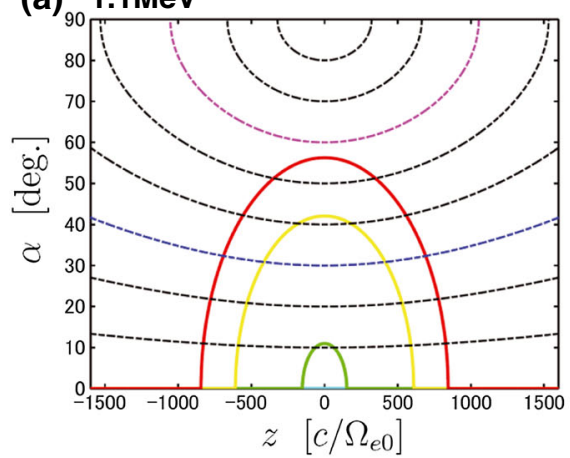

(c) $3.1 \mathrm{MeV}$

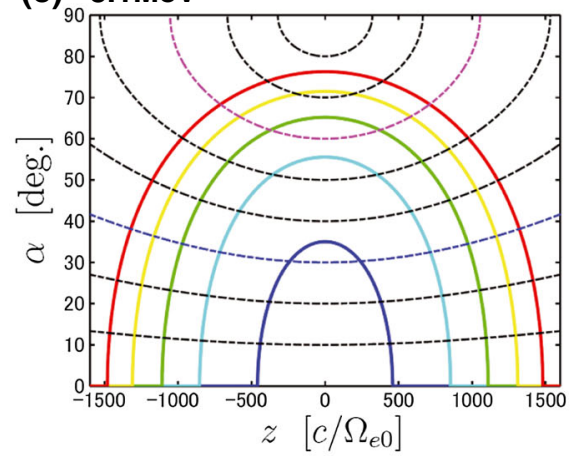

(e) $5.1 \mathrm{MeV}$

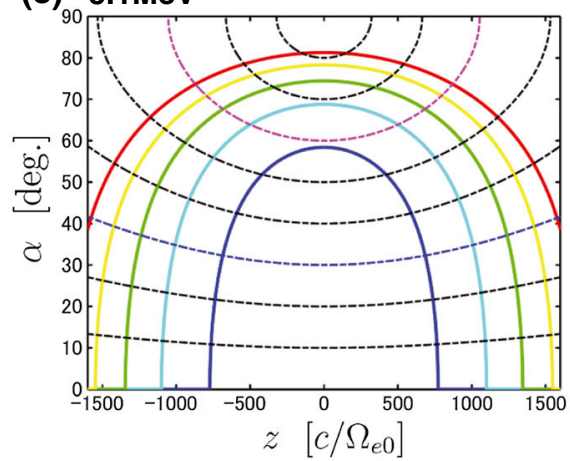

(b) $2.1 \mathrm{MeV}$

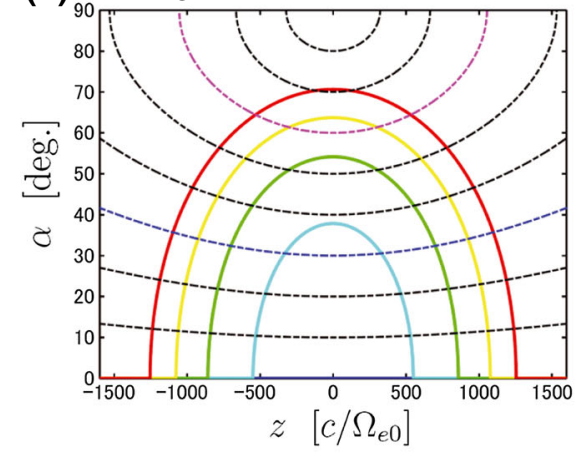

(d) $4.1 \mathrm{MeV}$

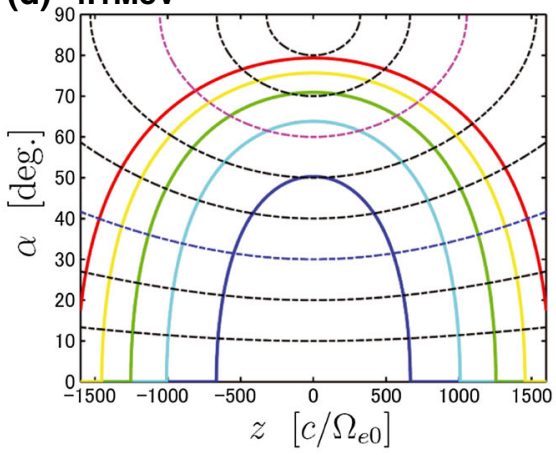

(f) $6.1 \mathrm{MeV}$

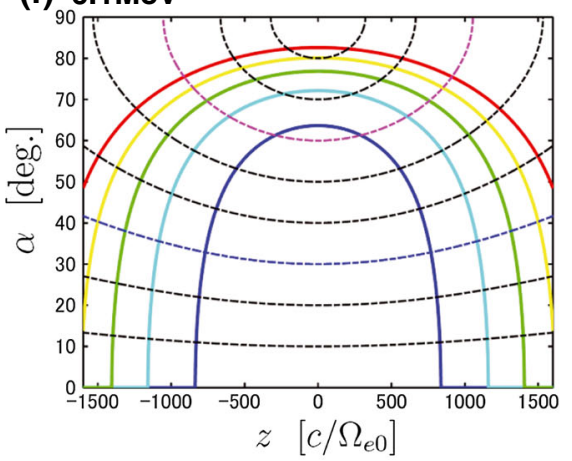

Figure 3. Pitch angles of relativistic electrons satisfying the anomalous cyclotron resonance condition with EMIC waves with different frequencies indicated by red, yellow, green, cyan, and blue solid lines corresponding to wave frequencies $2.80,2.53,2.25,1.96$, and $1.70 \mathrm{~Hz}$, respectively. The waves propagate along the parabolic magnetic field specified by the coefficient $a=3.0 \times 10^{-7} \Omega_{e 0}^{2} / c^{2}$. The black dashed lines represent the adiabatic trajectories of electrons for the equatorial pitch angles $10,20,40,50,70,80^{\circ}$, while the adiabatic trajectories for 30 and $60^{\circ}$ are plotted in blue and magenta dashed lines, respectively. The resonance conditions are plotted for different energies of electrons: (a) 1.1, (b) 2.1, (c) 3.1, (d) 4.1, (e) 5.1 , and (f) $6.1 \mathrm{MeV}$.

Since the resonance condition is also determined by energies of electrons, the resonance curves are plotted for different energies (a) $1.1 \mathrm{MeV}$, (b) $2.1 \mathrm{MeV}$, (c) $3.1 \mathrm{MeV}$, (d) $4.1 \mathrm{MeV}$, (e) $5.1 \mathrm{MeV}$, and (f) $6.1 \mathrm{MeV}$ in each panel of Figure 3. We assume a wave packet as illustrated in Figure 1. The wave packet of the EMIC triggered emission has the wave front with the frequency $1.7 \mathrm{~Hz}$ and the wave tail with the frequency $2.8 \mathrm{~Hz}$. Namely, the red line refers to the wave tail of $2.8 \mathrm{~Hz}$, while the blue line refers to the wave front of 1.7 $\mathrm{Hz}$ in Figure 3. The green line corresponds to the middle part of the wave packet. The resonant electrons pass through the wave packet from the tail to the front, seeing the wave frequency decreasing from 2.8 to $1.7 \mathrm{~Hz}$.
[10] When electrons are out of resonance, they follow adiabatic bounce motions with pitch angle variations given by

$$
\sin \alpha=\sqrt{\frac{B_{0}(z)}{B_{\mathrm{EQ}}}} \sin \alpha_{\mathrm{EQ}},
$$

In Figure 3, the adiabatic orbits are also plotted in black dashed lines for $\alpha_{\mathrm{EQ}}=10,20,40,50,70$, and $80^{\circ}$ and blue and magenta dashed lines for 30 and $60^{\circ}$, respectively.

[11] Nonlinear wave trapping of resonant electrons takes place in the velocity phase space $(\zeta, \theta)$, where $\zeta$ is an angle between a perpendicular velocity $v_{\perp}$ of an electron and the 
wave magnetic field, and $\theta$ represents a difference between a parallel velocity $v_{\|}$and the resonance velocity $V_{R}$ converted to the unit of frequency, i.e., $\theta=k\left(v_{\|}-V_{R}\right)$. The dynamics of the trapped electrons is described by the following set of equations derived by OZ12.

$$
\frac{\mathrm{d} \zeta}{\mathrm{d} t}=-\theta
$$

and

$$
\frac{\mathrm{d} \theta}{\mathrm{d} t}=\omega_{t r}^{2}(\sin \zeta+S),
$$

where $S$ is the inhomogeneity factor given by

$$
S=-\frac{1}{\omega_{t r}^{2}}\left(s_{1} \frac{\partial \omega}{\partial t}+s_{2} V_{p} \frac{\partial \Omega_{e}}{\partial z}\right),
$$

and $\omega_{t r}$ is the trapping frequency given by

$$
\omega_{t r}=\sqrt{\frac{k v_{\perp} \Omega_{w}}{\gamma}},
$$

where $\Omega_{w}=e B_{w} / m_{e}$.

[12] Setting the second-order derivative of the phase angle $\zeta$ as zero, i.e., $\mathrm{d} \theta / \mathrm{d} t=-\mathrm{d}^{2} \zeta / \mathrm{d} t^{2}=0$ in (16), we obtain the second-order resonance condition

$$
\sin \zeta+S=0,
$$

whereas $\theta=0$ in (15), i.e., $v_{\|}=V_{R}$ is called the first-order resonance condition. When $|S|<1$, we can find two phase angles satisfying the condition. One is a stable equilibrium point, around which trapped electrons oscillate. The other is an unstable equilibrium point or a saddle point.

[13] The parameters $s_{1}$ and $s_{2}$ are given by

$$
\begin{gathered}
s_{1}=\left(\frac{V_{R}}{V_{g}}-1\right)^{2}, \\
s_{2}=\frac{\omega}{\Omega_{e}}\left(\frac{v_{\perp}^{2}-V_{R}^{2}}{2 V_{p}^{2}}+\frac{V_{R}^{2}}{V_{g} V_{p}}\right)+\frac{V_{R}}{\gamma V_{p}},
\end{gathered}
$$

and $V_{p}=\omega / k$. The group velocity is derived by $\mathrm{OZ} 12$ as

$$
V_{g}=\frac{2 c^{2} k}{\omega \sigma},
$$

where

$$
\sigma=\sum_{s} \frac{\omega_{p s}^{2}\left(2 \Omega_{s}-\omega\right)}{\Omega_{s}\left(\Omega_{s}-\omega\right)^{2}} .
$$

Since the velocity of a resonant electron is much greater than the group velocity of the wave packet, we can assume $V_{R} \gg V_{g}$, and we have

$$
s_{1}=\frac{V_{R}^{2}}{V_{g}}=\frac{\left(\omega \Omega_{e} \sigma\right)^{2}}{4 \gamma^{2} k^{4} c^{4}} .
$$

We consider the necessary condition for nonlinear wave trapping near the magnetic equator neglecting the spatial gradient of the magnetic field in the inhomogeneity factor, which is rewritten as

$$
S=-\frac{s_{1}}{\omega_{t r}^{2}} \frac{\partial \omega}{\partial t} .
$$

To satisfy the second-order resonance condition, we need the condition $|S|<1$. Setting $S=-1$ in (17), substituting (13), (18), and (24) into (25), and solving for $\Omega_{w}$, we obtain the threshold amplitude $\Omega_{t h}$ for the nonlinear trapping of relativistic electrons by an EMIC triggered emission as

$$
\tilde{\Omega}_{t h}=\frac{\sigma^{2} \Omega_{e 0}^{3}}{4 \gamma \Pi_{c}^{5 / 2} \omega^{1 / 2}}\left[1-\frac{1}{\gamma^{2}}\left(1+\frac{\Omega_{e 0}^{2}}{\omega \Pi_{c}}\right)\right]^{-1 / 2} \frac{\partial \tilde{\omega}}{\partial \tilde{t}},
$$

where $\tilde{\Omega}_{t h}=\Omega_{t h} / \Omega_{e 0}, \tilde{\omega}=\omega / \Omega_{e 0}$, and $\tilde{t}=t \Omega_{e 0}$. The nonlinear wave trapping of resonant electrons becomes possible at the equator, when $S>-1$, which is rewritten as a condition of the wave amplitude $B_{w}$ for the nonlinear trapping as

$$
B_{w}>\tilde{\Omega}_{t h} B_{\mathrm{EQ}} .
$$

[14] Resonant electrons trapped by a wave packet are guided along the resonance velocity which increases as they move away from the equator. Trapped electrons are gradually detrapped because the gradient of the magnetic field as expressed by the second term in (17) increases to make $|S|$ greater. Once $|S|>1$, all electrons are detrapped. The changes of the wave amplitude $\Omega_{w}$ and the wave number $k$ in (18) also contribute to the variation of $|S|$. The wave can grow through propagation from the equator to higher latitudes. Both $B_{w}$ and the gradient of the magnetic field increases, making the frequency sweep rate less important in controlling the inhomogeneity factor $S$. It is also noted that the threshold amplitude becomes smaller for higher-energy electrons because of the dependency on $\gamma$ in (26). Highly energetic electrons with appropriate ranges of pitch angles shown in Figures 3c-3f, can easily be trapped near the equator by EMIC triggered emissions even with relatively small wave amplitudes.

\section{Test Particle Simulations With a Simplified Wave Packet}

[15] With the simple wave packet model defined by the wave front and tail assumed in OZ12, we perform test particle simulations with different energies (a) 1.1, (b) 2.1, (c) 3.1 , (d) 4.1 , (e) 5.1 , and (f) $6.1 \mathrm{MeV}$, corresponding to the six cases presented in Figure 3. We assume the wave front with a wave frequency $1.7 \mathrm{~Hz}\left(0.46 \Omega_{H 0}\right.$ or $\left.2.51 \times 10^{-4} \Omega_{e 0}\right)$ at $z=1000 c / \Omega_{e 0}$ and the wave tail with a wave frequency $2.8 \mathrm{~Hz}\left(0.76 \Omega_{H 0}\right.$ or $\left.4.25 \times 10^{-4} \Omega_{e 0}\right)$ at $z=0 c / \Omega_{e 0}$. The wave frequencies between the wave front and tail are linearly interpolated in space, while the wave amplitude is assumed constant at $B_{w}=0.009 B_{\mathrm{EQ}}$, which corresponds to $2.2 \mathrm{nT}$ for $L=4.27$. In Figure 4, we plot time histories of pitch angles $\alpha$, the distribution $F$ of the resonant electrons as a function of an equatorial pitch angle $\alpha_{\mathrm{EQ}}$, and $D=\left(v_{\|}-V_{R}\right) / V_{t r}$, where $V_{t r}$ is the trapping velocity given by

$$
V_{t r}=2 \frac{\omega_{t r}}{k}=2 \sqrt{\frac{v_{\perp} \Omega_{w}}{\gamma k}}
$$

which is derived by OZ12. The wave model and other conditions are the same as assumed in the simulation shown in Figure 5e of OZ12, and the physical parameters are listed in Table 1. 
(a)

$\alpha$ [deg.]

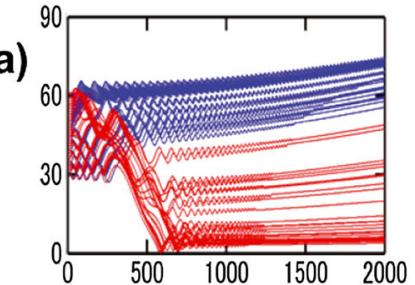

(b)

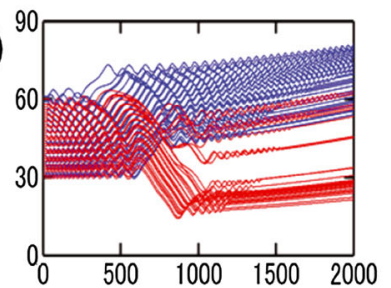

(c)

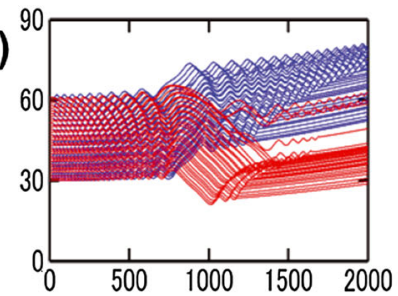

(d)

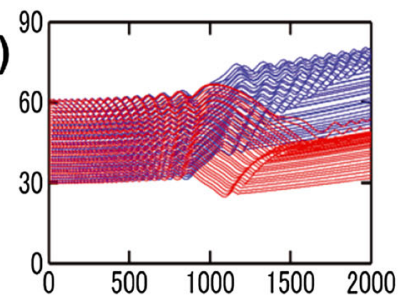

(e)

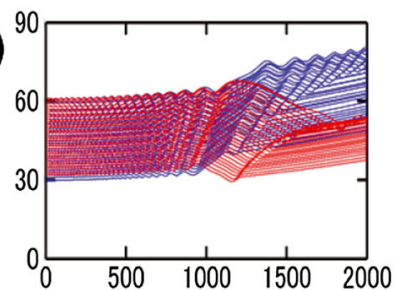

(f)

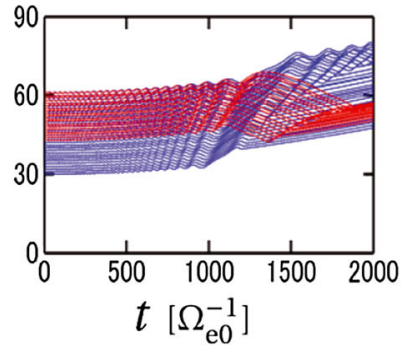

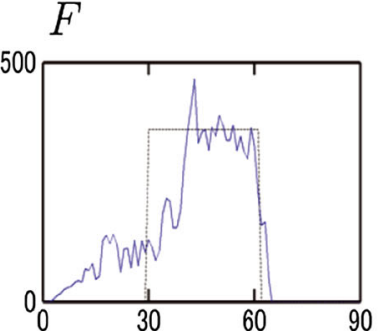
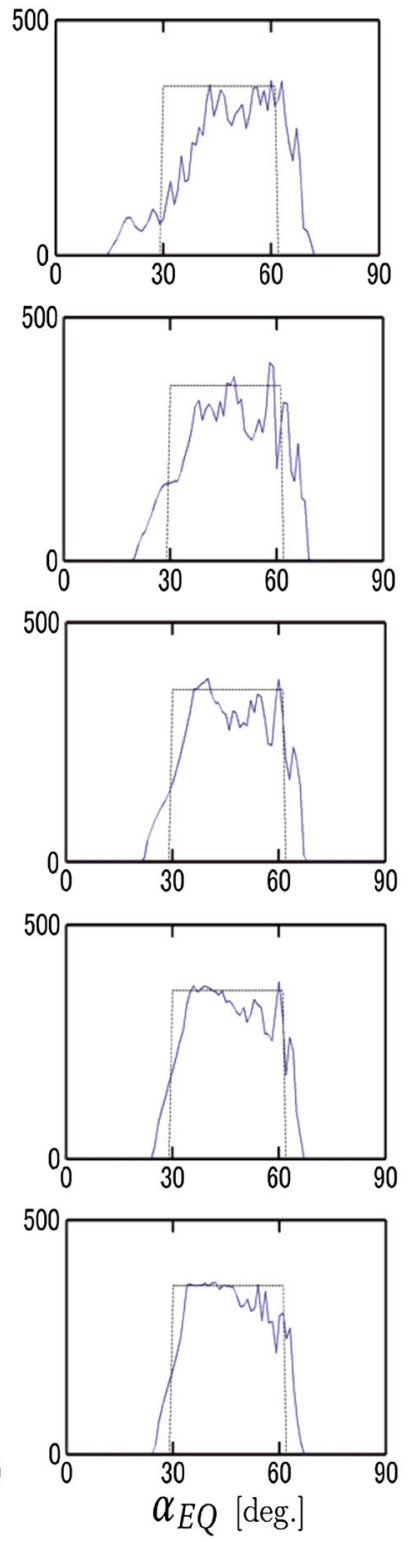

$D$
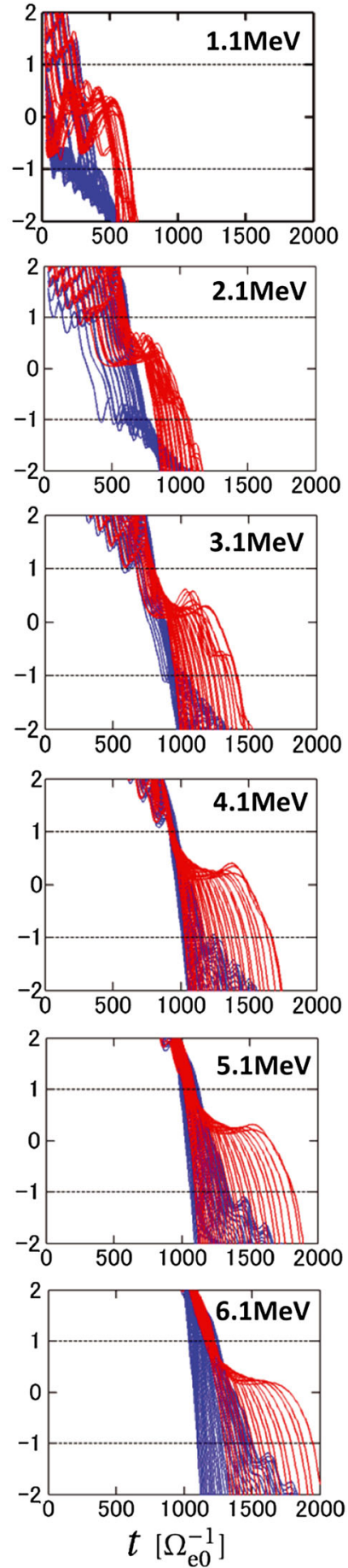

Figure 4. Trajectories of resonant electrons in (left) pitch angles $\alpha$ interacting with an EMIC wave packet with a rising frequency from 1.7 to $2.8 \mathrm{~Hz}$, (middle) distributions $F$ of electrons as functions of the equatorial pitch angle $\alpha_{\mathrm{EQ}}$ and the initial distributions in dotted lines, and (right) trajectories in $D=\left(v_{\|}-V_{R}\right) / V_{t r}$. The energies of resonant electrons are (a) 1.1 , (b) 2.1 , (c) 3.1 , (d) 4.1 , (e) 5.1 , and (f) $6.1 \mathrm{MeV}$.

[16] We inject electrons at $z=-10 c / \Omega_{e 0}$ with positive parallel velocities. In the left and right columns of Figure 4, we plot trajectories of specific electrons showing the most significant changes of pitch angles at different initial pitch angles from 30 to $60^{\circ}$ with an interval of $1^{\circ}$. At each pitch angle, we trace 360 particles with different gyrophases with an interval of $1^{\circ}$. If the maximum decrease in pitch angle is $5^{\circ}$ or more, it is plotted in red. If the maximum increase in pitch angle is $1^{\circ}$ or more, it is plotted in blue. When electrons satisfy $-1<D<1$, it means that the electrons can be 
(a) $\alpha$ [deg.]

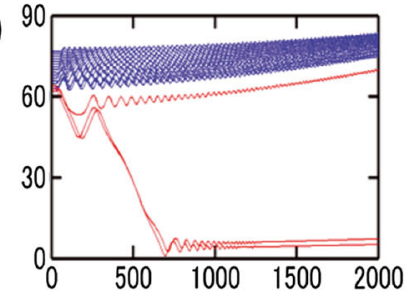

(b)

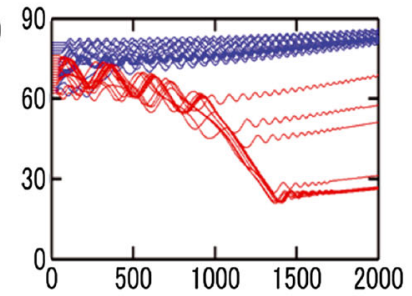

(c)

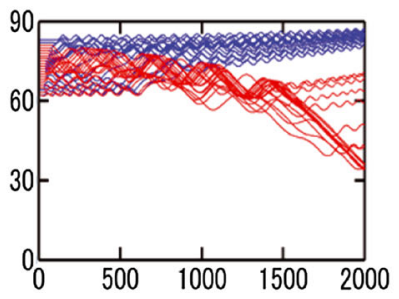

(d)

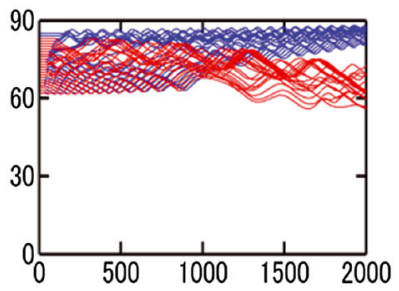

(e)

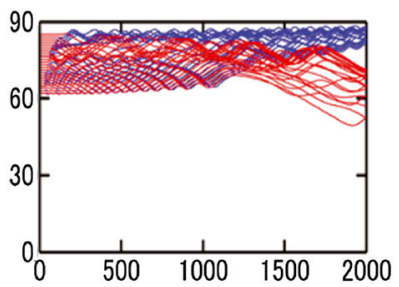

(f)

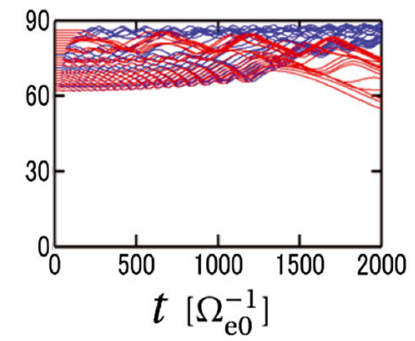

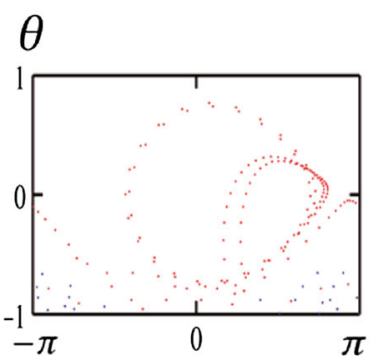
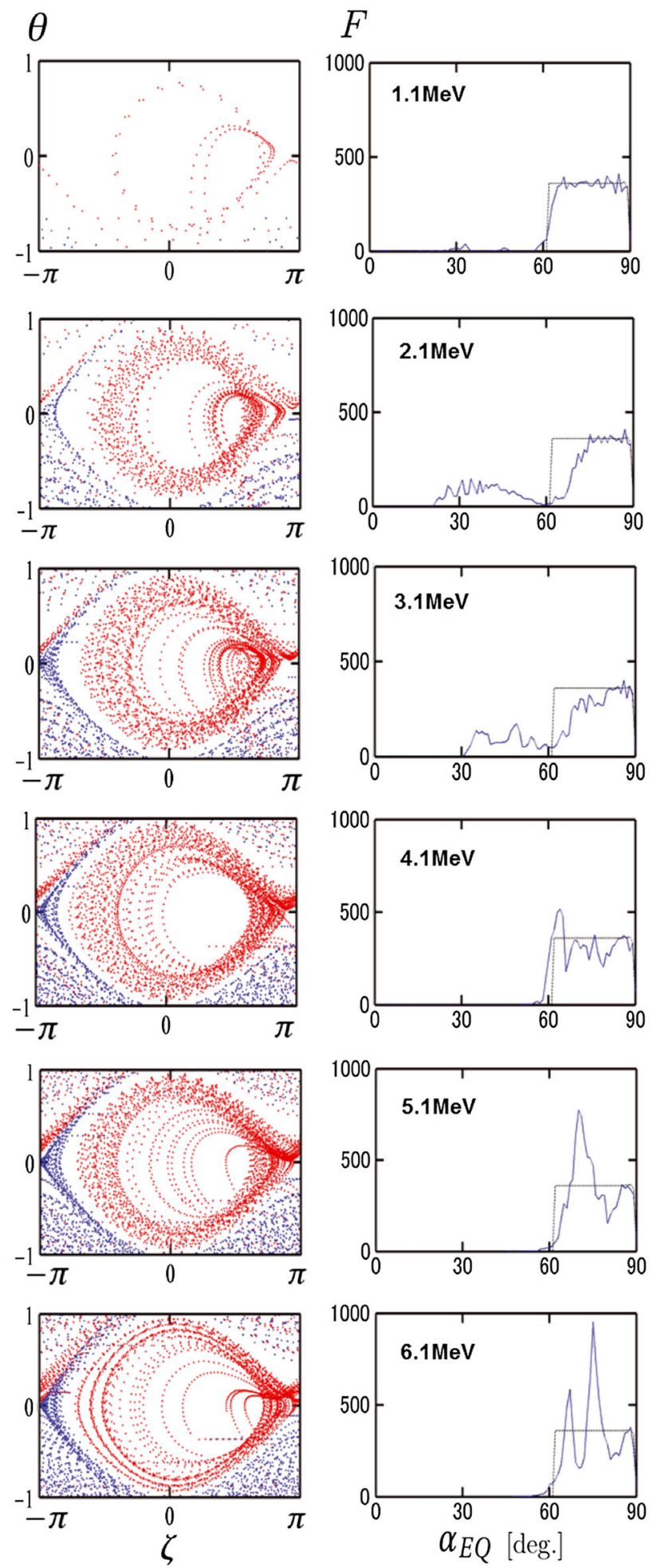

Figure 5. Trajectories of resonant electrons (left) in pitch angle $\alpha$, (middle) in the phase space $(\theta, \zeta)$, and (right) distributions $F$ similar to Figure 4 (middle) for the initial pitch angles from 61 to $89^{\circ}$.

trapped by the EMIC waves. We plot $D= \pm 1$ with black dashed lines for reference. As shown in Figure 4a, electrons with energy of $1.1 \mathrm{MeV}$ satisfy $-1<D<1$ soon after they move into the tail of the wave packet from the equator. The frequency at the wave tail is $2.80 \mathrm{~Hz}$ and that of the wave front is $1.70 \mathrm{~Hz}$. Some of the electrons are trapped by the wave potential and guided to pitch angles close to zero, as shown by the red trajectories in Figure 4a. The wave frequency seen from the trapped resonant electrons decreases from $2.80 \mathrm{~Hz}$ (red curve in Figure 3a) to lower frequencies, resulting in faster pitch angle scattering into the loss cone in short distances. With regard to $2.1 \mathrm{MeV}$ electrons, 
however, it takes a little time for $D$ to satisfy the trapping condition; the electrons are still trapped by the waves and scattered to about $20^{\circ}$ pitch angle. This is because the resonance curves are located farther away from the equator. For 3.1 and $4.1 \mathrm{MeV}$ electrons, it takes more time for $D$ to satisfy $-1<D<1$, and the electrons are only scattered to about $25^{\circ}$. For those 5.1 and $6.1 \mathrm{MeV}$ energy electrons, almost no trapping takes place. These changes of the trapping time can be explained by the change of $S$ value given by (17). When the energy becomes large, the $\gamma$ also increases. Thus $\omega_{t r}$ becomes small, making $|S|$ larger. It is also noted that the resonance condition is satisfied by the higher-energy electrons only at some distance away from the equator as shown in Figures 3e and 3f. Therefore, we find the longer adiabatic motions before the resonance for the higher-energy electrons. When the resonance points are located away from the equator, the inhomogeneity factor $|S|$ increases because of the gradient of the magnetic field. The trapping condition $|S|<1$ only holds for a short period of time. Therefore, higher-energy electrons are trapped only for a shorter time than lower-energy electrons.

[17] We now study dynamics of relativistic electrons with high pitch angles at the magnetic equator. Figure 3 shows that relativistic electrons with high pitch angles $\left(>60^{\circ}\right)$ can interact with EMIC waves at higher frequencies. Especially highly energetic electrons $(>4 \mathrm{MeV})$ can interact with a wide range of frequencies. We have conducted another test particle simulation with higher equatorial pitch angles $\left(>60^{\circ}\right)$ using the same wave packet model presented above. We show the simulation results in Figure 5. The energies of the electrons are set as (a) 1.1, (b) 2.1, (c) 3.1, (d) 4.1, (e) 5.1, and (f) $6.1 \mathrm{MeV}$, and the pitch angles are from 61 to $89^{\circ}$, with an interval of $1^{\circ}$. For all the cases, the trapping of the resonant electrons takes place, as we can confirm in the middle column of Figure 5 showing the phase plots in the $\zeta-\theta$ plane. For $1.1 \mathrm{MeV}$ electrons, although they can be scattered down to almost $0^{\circ}$, the trapping is not so efficient. Electrons at 2.1 and $3.1 \mathrm{MeV}$ are very effectively scattered to about $30^{\circ}$. These electrons from 30 to $60^{\circ}$ can interact with wave packet again near the equator and scattered to much lower pitch angles by the nonlinear trapping as we have seen in Figure 4. The electrons at 4.1, 5.1, and 6.1 MeV in Figures 5d, 5e, and 5f are scattered to about $60^{\circ}$. Theses electrons can also be scattered to lower pitch angles through repeated resonances with the wave packet through the bounce motion between the mirror points. As we can confirm from Figure 5, electrons with higher energies and higher pitch angles can also be trapped by the EMIC triggered emissions. Noting that $1000 \Omega_{e 0}^{-1}$ corresponds to $23 \mathrm{~ms}$, we find that the time scales of the precipitations are much less than a second.

\section{Simulations of Relativistic Electron Precipitation}

[18] As demonstrated in Figures 3, 4, and 5, most of the relativistic electrons can get into resonance with the wave packet of an EMIC triggered emission because of the risingtone frequency and the increasing magnetic field as they move away from the magnetic equator. We perform test particle simulations with a large number of relativistic electrons trapped in the magnetic field (7). In contrast to the simple model with injection of relativistic electrons from the equator for a single passage through the wave packet presented in the previous section, we inject electrons at different positions and follow their motions for a much longer time period of many bounce motions between the mirror points, while the wave packets of the EMIC triggered emissions are generated based on a more realistic physical model described below. To distribute trapped electrons in space as the initial condition, we calculate the position of the mirror points $\pm z_{m}$ for an electron with pitch angle $\sim 6^{\circ}$ which is assumed as the loss cone angle for $L=4.27$ [Ebihara and Ejiri, 2003]. The distance of the mirror points $z_{m}$ is given by

$$
z_{m}=\frac{1}{\sqrt{a} \tan \alpha_{\mathrm{EQ}}} .
$$

With the parabolic coefficient $a=3.0 \times 10^{-7} \Omega_{e 0}^{2} / c^{2}$ and $\alpha_{\mathrm{EQ}}=6^{\circ}$, we have $z_{m}=1.7371 \times 10^{4} \mathrm{c} / \Omega_{e 0}$. According to this result, we assume the boundary of the simulation field as $2 \times 10^{4} c / \Omega_{e 0}$. We compute the wave amplitude $B_{w}$, wave frequency $\omega$, wave number $k$, and wave phase $\psi$ of EMIC waves at each grid point. We assume the EMIC triggered emissions are generated at the equator and propagate to the higher-latitude regions according to the wave equations [Omura et al., 2010]:

$$
\frac{\partial B_{w}}{\partial t}+V_{g} \frac{\partial B_{w}}{\partial z}=-\frac{\mu_{0} V_{g}}{2} J_{E}
$$

where $\mu_{0}$ and $J_{E}$ are the magnetic permeability in vacuum and a component of the resonant current parallel to the wave electric field $E_{w}$, respectively.

$$
\frac{\partial \omega}{\partial t}+V_{g} \frac{\partial \omega}{\partial z}=0
$$

We solve the wave equations for the wave amplitude and frequency to reproduce the EMIC wave packet which propagates from the equator to the higher-latitude region.

[19] Time evolution of the wave amplitude and frequency of the EMIC triggered emissions at the equator is described by a set of equations called EMIC chorus equations [Omura et al., 2010]. For simplicity, however, we assume that the wave amplitude is constant throughout the wave generation process at the equator and propagation away from the equator. We use only one of the chorus equations to find the evolution of the wave frequency at the equator. Namely, the frequency variation is reproduced by integrating

$$
\frac{\partial \omega}{\partial t}=\frac{2 V_{\perp 0}}{5 V_{p}}\left(1-\frac{V_{R H}}{V_{g}}\right)^{-2} \omega \Omega_{H 0} \frac{B_{w}}{B_{\mathrm{EQ}}},
$$

where $V_{R H}=\left(\omega-\Omega_{H 0}\right) / k$ is the resonance velocity of a proton at the equator. We assume that the EMIC triggered emission is generated by the energetic protons whose average velocity is $V_{\perp 0}$. While the frequency is increasing, the wave amplitude is assumed constant. Once the frequency reaches the maximum frequency of the rising-tone emission, the emission is terminated by setting the wave amplitude $B_{w}=0$.

[20] Since the frequency sweep rate $\partial \omega / \partial t$ is determined by the wave amplitude at the equator, we can calculate the 
inhomogeneity factor $S$ for the resonant relativistic electrons to check if the nonlinear trapping of them are possible or not. Substituting (32) into (25), we obtain

$$
\begin{aligned}
S= & -\frac{V_{\perp 0}}{10 \gamma c}\left[1-\frac{1}{\gamma^{2}}\left(1+\frac{\Omega_{e 0}^{2}}{\omega \Pi_{c}}\right)\right]^{-1 / 2}\left(\frac{\sigma \Omega_{e 0}}{\Pi_{c}}\right)^{2} \\
& \cdot\left[1+\frac{\sigma\left(\Omega_{H 0}-\omega\right)}{2 \Pi_{c}}\right]^{-2} \frac{m_{e}}{m_{H}},
\end{aligned}
$$

where $m_{H}$ is the proton rest mass. When $|S|<1$, nonlinear trapping of electrons becomes possible at the equator, and the pitch angles of trapped resonant electrons change according to the following equation derived by OZ12.

$$
\frac{\mathrm{d} \alpha}{\mathrm{d} t}=\frac{\Omega_{w}}{\gamma} S+\frac{\nu_{\perp}}{2 \Omega_{e}} \frac{\partial \Omega_{e}}{\partial z},
$$

where the first term on the right-hand side dominates near the equator.

[21] It is noted that the dependency of $S$ on the wave amplitude disappears, and the controlling parameters are $V_{\perp 0}$ of the energetic protons and the energy of a resonant electron specified by $\gamma$. We have plotted $S$ as functions of the frequency $\omega$ for different energies $1-6 \mathrm{MeV}$ in different colors in Figure 2b. The limit of nonlinear trapping $S=-1$ is indicated by a black dashed line. Although the first-order resonance condition gives the minimum resonance energy for the frequency ranges of the EMIC wave dispersion relation as shown in Figure 2a, the second-order resonance condition with $|S|<1$ gives limitations of the frequency ranges for the nonlinear trapping, i.e., the effective pitch angle scattering of resonant electrons.

[22] We assume a constant wave amplitude $B_{w}=$ $0.009 B_{\mathrm{EQ}}$ at the equator $z=0$ while the EMIC wave is being generated with the varying frequency according to one of the EMIC chorus equations (32) with the initial wave frequency $\omega=2.51 \times 10^{-4} \Omega_{e 0}(1.7 \mathrm{~Hz})$. When the rising frequency reaches to $\omega=4.25 \times 10^{-4} \Omega_{e 0}(2.8 \mathrm{~Hz})$, the wave amplitude is set to zero, corresponding to the end of the wave packet. The variations of the frequency and the wave amplitude are used as the boundary conditions in solving the wave equations (30) and (31), where we assume $J_{E}=0$. We list the input parameters in Table 1. Figures $6 \mathrm{a}$ and $6 \mathrm{~b}$ show the evolutions of the wave amplitude and frequency in space and time, respectively. As we can see from the figures, the wave frequency increases from the $\omega=2.51 \times 10^{-4} \Omega_{e 0}$ $(1.7 \mathrm{~Hz})$ to $\omega=4.25 \times 10^{-4} \Omega_{e 0}(2.8 \mathrm{~Hz})$ with the constant amplitude $B_{w}=9 \times 10^{-3} B_{\mathrm{EQ}}(2.2 \mathrm{nT})$. We also assume that the EMIC triggered emissions propagate to both northern and southern hemispheres. The frequency sweep rate observed at some distance away from the equator decreases because of the wave dispersion effect. Namely, the group velocity decreases substantially as the frequency increases from $2.51 \times 10^{-4} \Omega_{e 0}$ to $4.25 \times 10^{-4} \Omega_{e 0}$ as indicated by the gradient of the color contours in Figure $6 \mathrm{~b}$. By comparing the time scales of the frequency variations from $3.2 \times 10^{-4}$ $\Omega_{e 0}$ to $4.2 \times 10^{-4} \Omega_{e 0}$ at the equator and $z=200 \mathrm{c} / \Omega_{e 0}$, we can understand that the frequency sweep rate at the higherfrequency part is much decreased over the short distance from the equator. The sweep rate decreases through propagation, and the magnitude of the inhomogeneity factor $|S|$ decreases, making it possible for the lower-energy electrons (a)

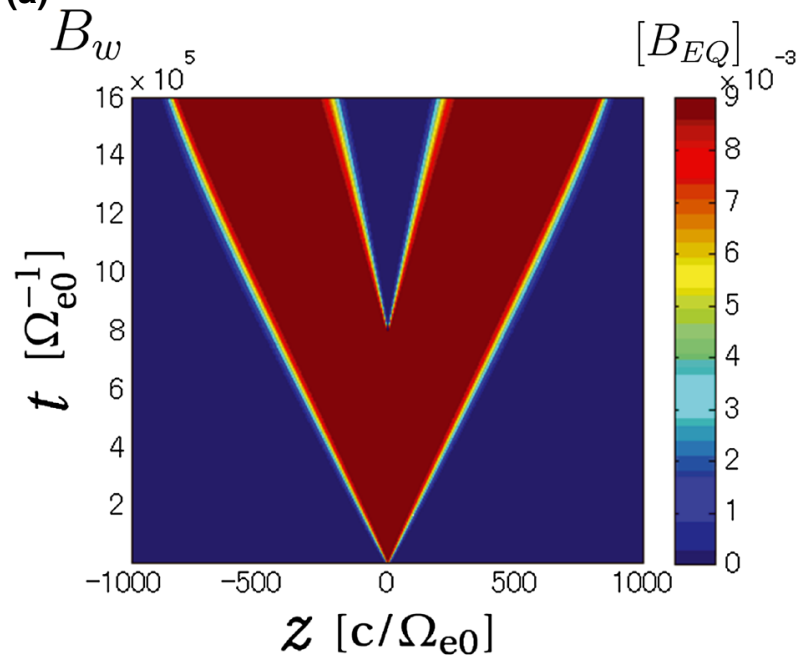

(b)

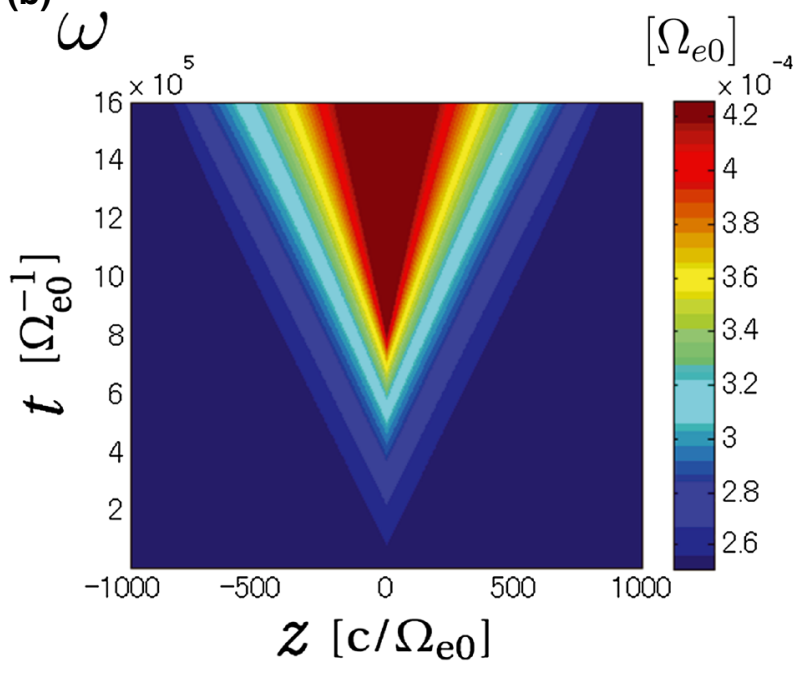

Figure 6. Variations of (a) the model wave amplitude $B_{w}$ and (b) frequency $\omega$ of the EMIC wave packets in space and time.

to be trapped. Although Figure $2 \mathrm{~b}$ shows that $S<-1$ for $1 \mathrm{MeV}$ electrons at the equator, we have $S>-1$ at higher latitudes. Since the higher-frequency part of the wave packet can resonate with $1 \mathrm{MeV}$ electrons at $z=200 \mathrm{c} / \Omega_{e 0}$ as shown in Figure 3a, trapping of $1 \mathrm{MeV}$ electrons is possible in the region away from the equator.

[23] If the wave frequency approaches to the cutoff frequency of the EMIC wave dispersion relation, the wave is either to be reflected or converted to the R-mode in the oblique propagation. In this simulation, 8,000,000 time steps are followed, and the wave front spreads to about $845 c / \Omega_{e 0}$, which is still before the wave front reaches the cutoff point. Similarly, we assume a backward wave packet, which propagates in the negative $z$ direction symmetrically with the forward wave packet propagating in the positive $z$ direction.

[24] As the initial condition, we distribute electrons at different positions between the mirror points $\pm z_{m}$ along the field line by using the uniform random numbers. When the equatorial pitch angle $\alpha_{\mathrm{EQ}}$ and a position $z$ of an 
electron is determined, we calculate the pitch angle $\alpha$ at $z$ by (7) and (14). Then, we can set $v_{\|}$and $v_{\perp}$ with different gyrophases.

\subsection{Case 1: $\alpha_{\mathrm{EQ}}=30-60^{\circ}$}

[25] We perform test particle simulations of interaction between EMIC triggered emissions and relativistic electrons with various pitch angles, phase angles, energies, positions, as well as directions. The pitch angles are from 30 to $60^{\circ}$ and phase angles of the perpendicular velocities are from 0 to $360^{\circ}$ with an interval of $1^{\circ}$. We set the initial energies of electrons from $300 \mathrm{keV}$ to $3.1 \mathrm{MeV}$ with an interval of $100 \mathrm{keV}$ and distribute them to both positive and negative $z$ directions for interaction with the pair of EMIC wave packets. The total number of relativistic electrons we used in simulation is 668,160 . Other input parameters are based on the Cluster observation [Pickett et al., 2010; Omura et al., 2010] as listed in Table 1.

[26] We show simulation results in Figure 7. Figure 7a shows distributions $F$ of the resonant electrons of different energy ranges as functions of an equatorial pitch angle $\alpha_{\mathrm{EQ}}$ which is calculated by (14). The dashed lines show the initial distribution, and solid lines show the distribution of the relativistic electrons after the interaction with the pair of EMIC triggered emissions. Electrons which have been scattered into the loss cone are excluded from the distribution. While some of the electrons are scattered to higher pitch angles, a substantial amount of electrons are transported to lower pitch angles by the nonlinear wave trapping. We count the number of electrons which fall into the loss cone during each time interval of $\Delta T=40 \Omega_{e 0}^{-1}$ and plot its time variation in Figure $7 \mathrm{~b}$. We classified the energy ranges $0.3-0.9,0.9-1.5$, $1.5-2.1,2.1-2.7$, and $2.7-3.3 \mathrm{MeV}$ by blue, green, magenta, black, and cyan, respectively. The numbers attached to the lines in Figure $7 \mathrm{~b}$ also indicate the energy ranges in $\mathrm{MeV}$. We also show total number and percentage of electrons that are scattered into the loss cone after the interaction with the EMIC triggered emissions in Figure $7 \mathrm{~b}$.

[27] We call the pair of EMIC wave packets used in this simulation as Emission 1. We can understand from this Figure $7 \mathrm{~b}$ that a substantial amount of electrons are scattered into the loss cone efficiently in a short time. Just with a single emission, Emission 1, 51.7\% of the initial electrons are scattered into the loss cone.

[28] The interaction between the resonant electrons and the EMIC waves takes place at the tail of the wave packet, and the electrons come out of the wave packet from the front with velocities much greater than the group velocity of the wave. At first, the packet of the wave is short, and since the frequency is growing up at the equator, the time variation of the frequency is not large. Therefore, some time is needed until the nonlinear trapping takes place, and there are few numbers of electrons that fall into the loss cone. The frequency at the tail of the wave packet grows and becomes large as time goes on, as well as the frequency sweep rate. Moreover, the wave also spreads away from the equator, and the wave packet becomes longer. Therefore, the trapping of the electrons becomes efficient. The trapped resonant electrons are scattered to lower pitch angles and effectively guided into the loss cone.

[29] Checking for the numbers of electrons falling into the loss cone in different energy ranges, we find that the
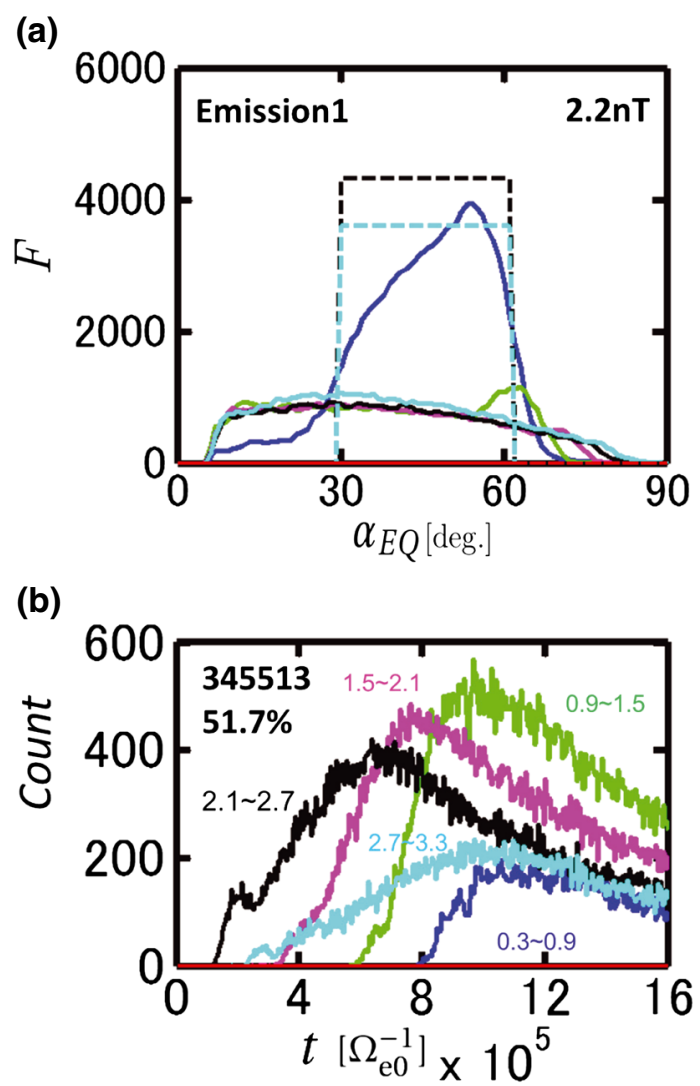

Figure 7. (a) Distribution functions $F$ of the resonant electrons of different energy ranges in the equatorial pitch angle $\alpha_{\mathrm{EQ}}$ for the initial values of $\alpha_{\mathrm{EQ}}=30-60^{\circ}$. The dashed lines indicate the initial distribution functions. (b) Numbers of test electrons falling into the loss cone over every time interval $\Delta T=40 \Omega_{e 0}^{-1}$ due to the interaction with the first pair of the wave packets of EMIC triggered emissions. The functions $\mathrm{F}$ and precipitation counts of electrons in energy ranges of $0.3-0.9,0.9-1.5,1.5-2.1,2.1-2.7$, and 2.7-3.3 $\mathrm{MeV}$ are plotted by blue, green, magenta, black, and cyan, respectively.

electrons of $0.3-0.9 \mathrm{MeV}$ are less scattered relatively. We refer to minimum resonance energy plotted in Figure 2a. The highest frequency of the triggered emission is $4.25 \times 10^{-4}$ $\Omega_{e 0}(2.8 \mathrm{~Hz})$, which is indicated by the blue dashed line in Figure $2 \mathrm{a}$. The lowest resonance energy is $0.5 \mathrm{MeV}$. The electrons in $0.3-0.5 \mathrm{MeV}$ cannot interact with the waves, and they remain in the distribution functions plotted in Figures 7a. Electrons in the range of $0.5-0.9 \mathrm{MeV}$ can be in resonance with the wave, but they cannot satisfy the second-order resonance condition at the equator as shown in Figure 2b. Only at some distance away from the equator, where the wave frequency sweep rate is much decreased because of the dispersion effect, the second-order resonance may take place, but it is only a short period of time because of increasing inhomogeneity of the magnetic field. The lower-energy electrons also interact with the higherfrequency part of the wave packet that appears later in time. Figure $2 \mathrm{~b}$ also shows that electrons less than $2 \mathrm{MeV}$ cannot be trapped at the equator. Trapping of the lower-energy electrons becomes possible at some distance away from the 
(a)

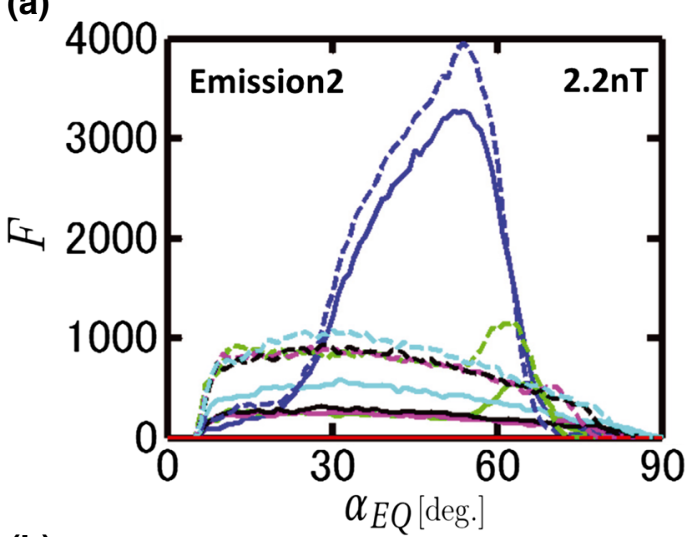

(b)

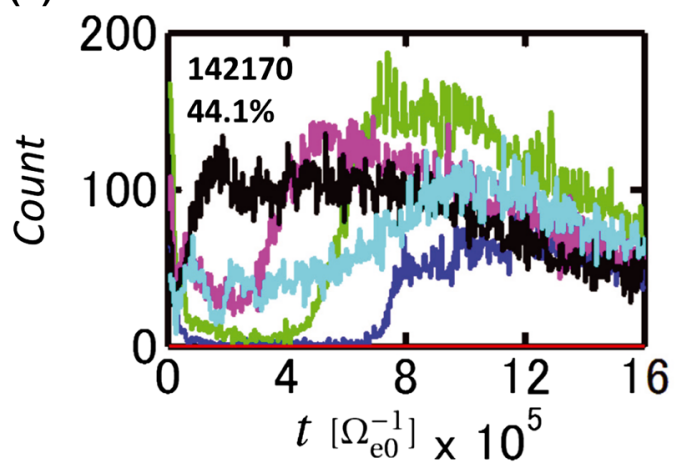

Figure 8. The same plots as Figure 7 for the interaction with the second pair of the wave packets of EMIC triggered emissions. Dashed lines in Figure 8a indicate the distribution functions after Emission 1 as plotted in solid lines in Figure 7(a).

equator with smaller frequency sweep rates due to the dispersion effect. Thus the precipitation of $0.3-0.9 \mathrm{MeV}$ only takes place much later in time at $8 \times 10^{5} \Omega_{e 0}^{-1}$ after the higher-frequency part of the emissions appears at the equator and propagates away from it. It is noted from Figure 6 that the rising frequency at the equator becomes higher than $0.33 \times 10^{-3} \Omega_{e 0}$ only after $6.0 \times 10^{5} \Omega_{e 0}^{-1}$.

[30] Electrons of 2.1-2.7 MeV are trapped very effectively, because they can be in resonance with the wave packet near the equator as shown in Figure 3. They can also be trapped at the equator, since $S>-1$ as shown in Figure $2 \mathrm{~b}$. The highly relativistic electrons greater than $2.7 \mathrm{MeV}$ is slightly delayed because the resonance points are located increasingly away from the equator for higher energies as shown in Figure 3. It takes time for the wave packet to propagate from the equator to the resonance points.

[31] After resonating with the wave packet, the electrons are bounced at the mirror points, and this time, they interact with the other wave packet in the opposite direction. Thus, bounce motions at the mirror points are repeated by the relativistic electrons that resonate with the EMIC wave packets several times near the equator. When the nonlinear trapping by the wave packets takes place, the electrons are scattered to lower pitch angles effectively.

[32] We set another emission that is generated at the equator and propagate away from the equator, interacting with the remaining electrons after Emission 1. We call the second pair of EMIC wave packets as Emission 2. The variation of the distribution functions and the precipitation are plotted in Figures $8 \mathrm{a}$ and $8 \mathrm{~b}$, respectively. The dashed lines in Figure 8a indicate distribution functions after interaction with Emission 1 as plotted in solid lines in Figure 7a. As a result of the second interaction, $44.1 \%$ of the remaining electrons are scattered into the loss cone. With Emissions 1 and 2 , the total $72.9 \%$ of the initial relativistic electrons are scattered into the loss cone. The relativistic electrons are trapped by the EMIC waves causing the relativistic microbursts. The time scale of REP corresponds to the time scales of the wave packet generation at the equator and propagation from it, namely $8-16 \times 10^{5} \Omega_{e 0}^{-1}$ corresponding to $20-40 \mathrm{~s}$.

\subsection{Case 2: $\alpha_{\mathrm{EQ}}=61-89^{\circ}$}

[33] Since we have obtained the result that the electrons with higher pitch angles can be trapped by EMIC triggered emissions as shown in Figure 5, we perform the simulation runs with the same wave packets and parameters as assumed in Case 1 except for pitch angle of electrons ranging from 61 to $89^{\circ}$. The simulation results are shown in Figures 9 and 10 . We trace total 605,520 relativistic electrons.
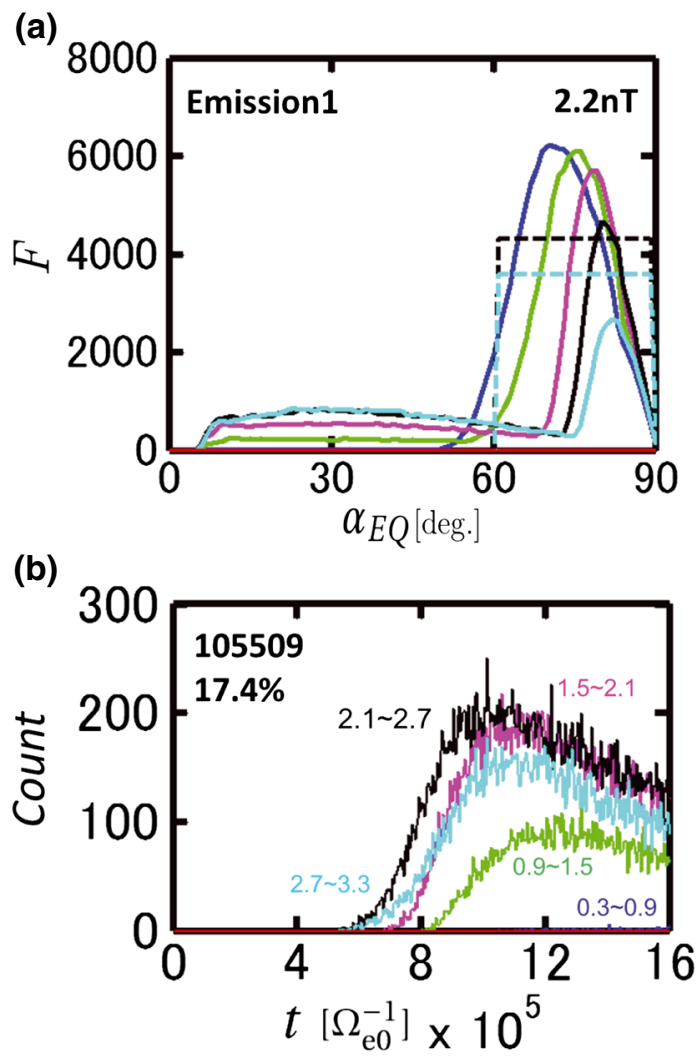

Figure 9. (a) Distribution functions $F$ of the resonant electrons in equatorial pitch angle $\alpha_{\mathrm{EQ}}$ for the initial values of $\alpha_{\mathrm{EQ}}=61-89^{\circ}$. (b) Numbers of test electrons falling into the loss cone over every time interval $\Delta T=40 \Omega_{e 0}^{-1}$ during the interaction with the first pair of the wave packets of EMIC triggered emissions. The functions $F$ and precipitation counts of electrons in energy ranges of $0.3-0.9,0.9-1.5$, 1.5-2.1, 2.1-2.7, and 2.7-3.3 MeV are plotted by blue, green, magenta, black, and cyan, respectively. 
(a)

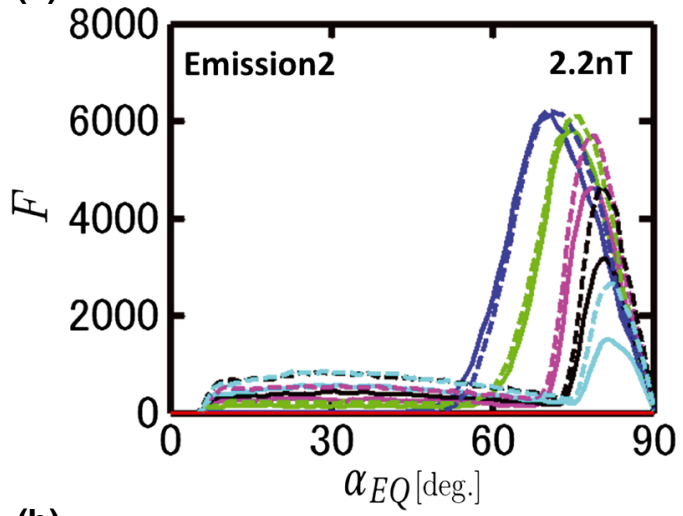

(b)

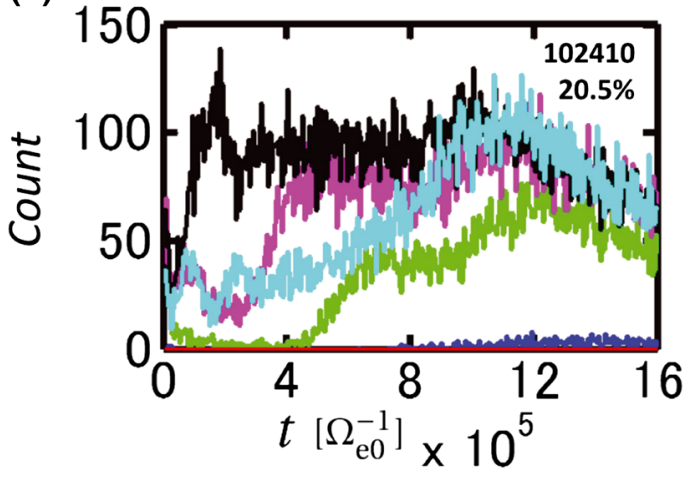

Figure 10. The same plots as Figure 9 for the interaction with the second pair of the wave packets of EMIC triggered emissions. Dashed lines in Figure 10a indicate the distribution functions after Emission 1 as plotted in solid lines in Figure 9(a).

We show the percentage of precipitated electrons in Figure 9b. In Figure 9, after interacting with Emission 1, $17.4 \%$ of the electrons are scattered into the loss cone. In Figure 10, after the interaction with Emission 2, 20.5\% of the remaining electrons are scattered into the loss cone. With these two emissions, the total $32.8 \%$ of the resonant electrons are scattered into the loss cone. Although this number is relatively small compared with the results from Figures 7 and 8 , we find that even the high pitch angle electrons can be scattered in pitch angles and precipitated into the loss cone through interaction with the EMIC triggered emissions repeated several times. From the simulation results from Figures 7, 8, 9, and 10, we can conclude that the EMIC triggered emissions have a high possibility of causing the relativistic electron microbursts.

\section{Summary and Discussion}

[34] We have studied the resonance condition between EMIC triggered emissions and relativistic electrons in an inhomogeneous magnetic field near the magnetic equator. Because of the variation of the wavelength $(2 \pi / k)$ of the EMIC wave packet, the resonance velocity $\Omega_{e} /(\gamma k)$ changes substantially. We have shown that most of the relativistic electrons get into the resonance, and some of them are trapped by the wave potential if the wave amplitude is greater than the threshold amplitude $\tilde{\Omega}_{t h}$ given by (26).
[35] Based on the nonlinear wave growth mechanism of EMIC triggered emissions, we have derived the formula (33) of the inhomogeneity factor $S$, by which we can judge the possibility of nonlinear trapping of relativistic electrons by the emissions at the equator. The formula indicates the direct relation between the perpendicular velocity of energetic protons generating EMIC triggered emissions and the energies and pitch angles of precipitated relativistic electrons.

[36] We performed test particle simulations to reproduce the relativistic microbursts. The model of the EMIC waves was developed based on the generation mechanism of the EMIC triggered emissions. We distributed a large number of electrons with different pitch angles, gyrophase angles, energies, and positions along the Earth's magnetic field. The electrons with wide ranges of energies and pitch angles can resonate with the EMIC triggered emission. The result shows that the EMIC wave causes effective pitch angle scattering and induces relativistic electron microbursts.

[37] We counted the precipitating electrons by checking the equatorial pitch angles at every time interval of $40 \Omega_{e 0}^{-1}$. As we can find in the trajectories of trapped resonant electrons guided to lower pitch angles shown in Figures 4 and 5 , the electrons increase pitch angles by a few degrees at the moment of detrapping from the wave potential after reaching the lowest pitch angles. Therefore, some of the electrons may have bounced back from the loss cone. More accurate counting of precipitating electrons could have been made by checking the pitch angles at mirror points or at the boundaries of the simulation system.

[38] As shown in Figure 5, electrons at high pitch angles can get into the resonance with the wave packet, because the resonance velocity of the high frequency part with the large $k$ becomes small. Electrons satisfying $V_{R}-V_{t r}<v_{\|}<$ $V_{R}+V_{t r}$ near the equator can be trapped and guided to higher $v_{\|}$, i.e., lower pitch angles (OZ12). Noting that the trapping velocity $V_{\text {tr }}$ is given by (28), we find that both large wave amplitude $\Omega_{w}$ and large $v_{\perp}$ make $V_{\text {tr }}$ large, and that the lower limit of the trapping velocity range $V_{R}-V_{\text {tr }}$ reaches zero. Since electrons near $90^{\circ}$ of pitch angle have $v_{\|} \sim 0$, they can be trapped by the nonlinear wave potential. The nonlinear trapping scatters the electrons to lower pitch angles as we find in Figures 9 and 10, which cannot be described by the quasi-linear theory, in which the diffusion rate for electrons at $90^{\circ}$ of pitch angle is typically the smallest due to lack of the cyclotron resonance.

[39] The present study can also provide a possible mechanism for runaway electrons found in a laboratory experiment of magnetic mirror trapped relativistic electrons, when shear Alfven waves are injected into the plasma [Wang et al., 2012].

[40] In this paper, we assumed wave packets with a constant amplitude. However, in the observations [Pickett et al., 2010; Omura et al., 2010] and the simulation [Shoji and Omura, 2011, 2012], the wave packets show modulation of the amplitudes. The amplitude modulations may cause detrapping of the trapped resonant electrons, making the pitch angle scattering less effective. On the other hand, the untrapped electrons can be trapped by a wave packet with an increasing wave amplitude as it moves away from the equator. EMIC triggered emissions in the proton band can also excite another EMIC wave in the helium band as reported by Shoji et al. [2011]. While the proton branch 


\section{OMURA AND ZHAO: RELATIVISTIC MICROBURSTS BY EMIC WAVES}

triggered emissions interact with a few $\mathrm{keV}$ protons, the helium branch triggered emissions interact with more energetic protons of a few hundred keV [Shoji and Omura, 2012]. As we can find in Figure 2, both first-order and second-order resonance conditions are satisfied by the helium branch triggered emissions. Test particle simulations on coherent pitch angle scattering by the helium band waves are left as a future study.

[41] In the present analysis, we assumed coherent waves in the parallel propagation, while the observed trigged emissions contain some frequency spread and they also propagate at oblique angles to the ambient magnetic field, making the polarization elliptic. The nonlinear trapping can be disrupted by the incoherent phase variation and the elliptical polarization. Finally the occurrences of EMIC triggered emissions have not been reported much. The overall contribution of the triggered emissions to the variation of the radiation belts should be evaluated quantitatively based on more cases of spacecraft observations near the magnetic equator.

[42] Acknowledgments. Computation in the present study was performed with the KDK system of Research Institute for Sustainable Humanosphere and Academic Center for Computing and Media Studies at Kyoto University as a collaborative research project. This work was supported by grants-in-aid 23340147 and 23224011 of the Ministry of Education, Science, Sports, and Culture of Japan. The authors thank Yuko Kubota for her useful questions and careful checking of the theoretical calculation.

[43] Robert Lysak thanks the reviewers for their assistance in evaluating this paper.

\section{References}

Anderson, R. R., and W. S. Kurth (1989), Discrete electromagnetic emissions in planetary magnetospheres, in Plasma Waves and Instabilities at Comets and in Magnetospheres, Geophys. Monogr. Ser., edited by Tsurutani, B. T., and H. Oya, p. 81-117, vol. 53, AGU, Washington, D.C.

Albert, J. M., and J. Bortnik (2009), Nonlinear interaction of radiation belt electrons with electromagnetic ion cyclotron waves, Geophys. Res. Lett., 36, L12110, doi:10.1029/2009GL038904.

Carson, B. R., C. J. Rodger, and M. A. Clilverd (2013), POES satellite observations of EMIC-wave driven relativistic electron precipitation during 1998-2010, J. Geophys. Res. Space Physics, 118, 232-243, doi:10.1029/2012JA017998.

Ebihara, Y., and M. Ejiri (2003), Numerical simulation of the ring current: Review, Space Sci. Rev., 105(1-2), 377-452.

Grison, B., O. Santolik, N. Cornilleau-Wehrlin, A. Masson, M. J. Engebretson, J. S. Pickett, Y. Omura, P. Robert, and R. Nomura (2013), EMIC triggered chorus emissions in cluster data, J. Geophys. Res. Space Physics, 118(3), 1159-1169, doi:10.1002/jgra.50178.

Johnston, W. R., and P. C. Anderson (2010), Storm time occurrence of relativistic electron microbursts in relation to the plasmapause, J. Geophys. Res., 115, A02205, doi:10.1029/2009JA014328.

Jordanova, V. K., J. Albert, and Y. Miyoshi (2008), Relativistic electron precipitation by EMIC waves from self-consistent global simulations, J. Geophys. Res., 113, A00A10, doi:10.1029/2008JA013239.

Kasahara, Y., Y. Miyoshi, Y. Omura, O. P. Verkhoglyadova, I. Nagano, I. Kimura, and B. T. Tsurutani (2009), Simultaneous satellite observations of VLF chorus, hot and relativistic electrons in a magnetic storm "recovery" phase, Geophys. Res. Lett., 36, L01106, doi:10.1029/ 2008GL036454.

Lauben, D. S., U. S. Inan, T. F. Bell, D. L. Kirchner, S. B. Hospodarsky, and J. S. Pickett (1998), VLF chorus emissions observed by Polar during the January 10, 1997 magnetic cloud, Geophys. Res. Lett., 25, 2995.
Lauben, D. S., U. S. Inan, T. F. Bell, and D. A. Gurnett (2002), Source characteristics of ELF/VLF chorus, J. Geophys. Res., 107, 1429, doi:10.1029/2000JA003019.

Liu, K., D. Winske, S. P. Gary, and G. D. Reeves (2012), Relativistic electron scattering by large amplitude electromagnetic ion cyclotron waves: The role of phase bunching and trapping, J. Geophys. Res., 117, A06218, doi:10.1029/2011JA017476.

Lorentzen, K. L., M. D. Looper, and J. B. Blake (2001), Relativistic electron microbursts during the GEM storms, Geophys. Res. Lett., 38, doi:10.1029/2001GL012926.

Miyoshi, Y., K. Sakaguchi, K. Shiokawa, D. Evans, J. Albert, M. Connors, and V. Jordanova (2008), Precipitation of radiation belt electrons by EMIC waves, observed from ground and space, Geophys. Res. Lett., 35, L23101, doi:10.1029/2008GL035727.

Omura, Y., Y. Katoh, and D. Summers (2008), Theory and simulation of the generation of whistler-mode chorus, J. Geophys. Res., 113, A04223, doi:10.1029/2007JA012622.

Omura, Y., M. Hikishima, Y. Katoh, D. Summers, and S. Yagitani (2009), Nonlinear mechanisms of lower-band and upper-band VLF chorus emissions in the magnetosphere, J. Geophys. Res., 114, A07217, doi:10.1029/2009JA014206.

Omura, Y., J. Pickett, B. Grison, O. Santolik, I. Dandouras, M. Engebretson, P. M. E. Decreau, and A. Masson (2010), Theory and observation of electromagnetic ion cyclotron triggered emissions in the magnetosphere, $J$ Geophys. Res., 115, A07234, doi:10.1029/2010JA015300.

Omura, Y., and Q. Zhao (2012), Nonlinear pitch angle scattering of relativistic electrons by EMIC waves in the inner magnetosphere, J. Geophys. Res., 117, A08227, doi:10.1029/2012JA017943.

Pickett, J. S., et al. (2010), Cluster observations of EMIC triggered emissions in association with Pc1 waves near Earth's plasmapause, Geophys. Res. Lett., 37, L09104, doi:10.1029/2010GL042648.

Rodger, C. J., T. Raita, M. A. Clilverd, A. Seppala, S. Dietrich, N. R. Thomson, and T. Ulich (2008), Observations of relativistic electron precipitation from the radiation belts driven by EMIC waves, Geophys. Res. Lett., 35, L16106, doi:10.1029/2008GL034804.

Sandanger, M. I., F. Sorras, M. Sorbo, K. Aarsnes, K. Oksavik, and D. S. Evans (2009), Relativistic electron losses related to EMIC waves during CIR and CME storms, J. Atmos. Sol. Terr. Phys., 71, doi:10.1016/ j.jastp.2008.07.006.

Santolik, O., D. A. Gurnett, J. S. Pickett, M. Parrot, and N. CornilleauWehrlin (2003), Spatio-temporal structure of storm-time chorus, J. Geophys. Res., 108, A71278, doi:10.1029/2002JA00979.

Shoji, M., and Y. Omura (2011), Simulation of electromagnetic ion cyclotron triggered emissions in the Earth's inner magnetosphere, J. Geophys. Res., 116, A05212, doi:10.1029/2010JA016351.

Shoji, M., and Y. Omura (2012), Precipitation of highly energetic protons by helium branch electromagnetic ion cyclotron triggered emissions, J. Geophys. Res., 117, A12210, doi:10.1029/2012JA017933.

Shoji, M., Y. Omura, B. Grison, J. Pickett, I. Dandouras, and M. Engebretson (2011), Electromagnetic ion cyclotron waves in the helium branch induced by multiple electromagnetic ion cyclotron triggered emissions, Geophys. Res. Lett., 38, L17102, doi:10.1029/ 2011 GL048427.

Spasojevic, M., L. Blum, E. A. MacDonald, S. A. Fuselier, and D. I. Golden (2011), Correspondence between a plasma-based EMIC wave proxy and subauroral proton precipitation, Geophys. Res. Lett., 38, L23102, doi:10.1029/2011GL049735.

Summers, D., B. Ni, and N. P. Meredith (2007a), Timescales for radiation belt electron acceleration and loss due to resonant waveparticle interactions: 1. Theory, J. Geophys. Res., 112, A04206, doi:10.1029/2006JA011801.

Summers, D., B. Ni, and N. P. Meredith (2007b), Timescales for radiation belt electron acceleration and loss due to resonant wave-particle interactions: 2. Evaluation for VLF chorus, ELF hiss, and EMIC waves, J. Geophys. Res., 112, A04207, doi:10.1029/2006JA011993.

Tsurutani, B. T., and E. J. Smith (1974), Postmidnight chorus: A substorm phenomenon, J. Geophys. Res., 79, 118.

Wang, Y., W. Gekelman, P. Pribyl, and K. Papadopoulos (2012), Scattering of magnetic mirror trapped fast electrons by a shear Alfvén wave, Phys. Rev. Lett., 108, 105002 